\title{
Context sensitivity of conditioned suppression following preexposure to the conditioned stimulus
}

\author{
DALE SWARTZENTRUBER and MARK E. BOUTON \\ University of Vermont, Burlington, Vermont
}

\begin{abstract}
Conditioned suppression in rats is often unaffected when the context (or set of background stimuli) is changed following conditioning. This suggests that responding to the conditioned stimulus (CS) can be relatively insensitive to the context in which the CS is presented. In two experiments, we examined whether sensitivity to contextual stimuli is affected by preexposure to the CS. In Experiment 1, when the CS was novel at the outset of conditioning, conditioned suppression was not affected when the context was changed following conditioning. However, when the CS had been preexposed, responding was weaker when extinction occurred outside of the conditioning context. In Experiment 2, responding was again sensitive to the test context, regardless of whether preexposure occurred in the conditioning context or in an alternate context. These results suggest that the extent to which responding is sensitive to context can depend on the conditioning history of the CS.
\end{abstract}

The role of contextual stimuli in controlling performance to conditioned stimuli (CSs) is an issue that has generated substantial interest. Although the response to a CS often depends on the subject's knowledge of the context in which the CS is presented (see Balsam \& Tomie, 1985 ), the context does not always play a strong role in controlling responding to a CS. For example, when a CS is tested outside of the conditioning context immediately following excitatory conditioning, conditioned responding is often unaffected (see, e.g., Bouton \& King, 1983; Bouton \& Peck, 1989; Grahame, Hallam, Geier, \& Miller, 1990; Hall \& Honey, 1989; Kaye, Preston, Szabo, Druiff, \& Mackintosh, 1987; Lovibond, Preston, \& Mackintosh, 1984; Swartzentruber \& Bouton, 1986). This suggests that, under some conditions, performance to a CS can be quite insensitive to contextual stimuli.

Recent research has examined several variables that may affect the degree to which responding to a CS is sensitive to the context in which it is presented. For example, Hall and Honey (1989) found that appetitive, as opposed to aversive, unconditioned stimuli (USs) establish conditioning that may be disrupted when the context is changed following conditioning (but see Bouton \& Peck, 1989; Kaye \& Mackintosh, 1990). Kaye and Mackintosh (1990), on the other hand, found that a context change enhanced responding to an aversively conditioned CS but did not affect responding to an appetitively conditioned CS. Thus,

This research was supported by National Science Foundation Grants BNS 86-07208 and BNS 89-08535 to M. E. Bouton and by a National Institute of Mental Health postdoctoral training Grant MH 09896 to D. Swartzentruber. We thank R. A. Rescorla for his comments on a draft of this manuscript. Address correspondence to D. Swartzentruber, who is now in the Department of Psychology, University of Pennsylvania, 3815 Walnut Street, Philadelphia, PA 19104. the type of US used may affect the degree of context sensitivity. Further, Hall and Honey (1990) found that aversive conditioning was disrupted by a change of context when only one conditioning trial, as opposed to multiple trials, was used during conditioning. Consequently, amount of training might be another influential variable. Although a summarizing principle has yet to emerge, it seems clear that simple conditioned responding is sensitive to the context in which the CS is presented in certain procedures and/or preparations.

Several findings from our own laboratory have encouraged the view that context sensitivity might also depend on the nature of the animal's prior experience with the CS. For example, although simple conditioned performance often generalizes strongly across contexts, $e x$ tinction performance is specific to its context. Sensitivity to context following extinction is revealed by "renewed" responding when the context is changed following extinction (Bouton \& Bolles, 1979; Bouton \& King, 1983; Bouton \& Peck, 1989; Bouton \& Swartzentruber, 1986). This suggests that the context may be more important following some types of learning as opposed to others.

Responding to simple excitatory CSs and CSs that have undergone both conditioning and extinction may be differentially sensitive to context for several reasons. First, a CS that has simply been conditioned evokes a response, whereas the conditioned and extinguished CS does not. Thus, one possibility is that contexts may have relatively little influence on excitatory CSs, but are important in controlling responding to CSs that are currently under the influence of extinction. Second, extinction always follows an episode in which the CS was conditioned. Extinction is therefore never the animal's first experience with the CS. It may be that a role for context emerges in situations in which a CS is associated with different outcomes in different phases of the experiment. 
The present experiments were designed to evaluate these possibilities. Specifically, for some rats, conditioning was the animals' first experience with the CS. For others, conditioning was preceded by nonreinforced exposure to the CS and was thus the animals' second experience with the CS. In order to evaluate the extent to which conditioned responding was sensitive to the context, animals from each treatment condition were tested in either the same context as that for conditioning or a different context. If the degree of context sensitivity is affected by the nature of the animal's history with the CS, we might find a greater role for context when conditioning follows CS exposure.

\section{EXPERIMENT 1}

Experiment 1 was designed to test whether conditioned responding is sensitive to the context when conditioning follows an initial phase of nonreinforced CS exposure. Four groups of rats received fear conditioning with a CS in Context A. Responding to the CS was then tested in either Context A or Context B. Prior to conditioning, two of these groups received exposure to the CS. If conditioning is more sensitive to the context following a phase of CS exposure, the identity of the testing context might influence the level of responding in the preexposed groups. We expected similar levels of suppression regardless of the test context for the groups that did not receive preexposure.

Nonreinforced preexposure to a CS has been shown to interfere with conditioning (see, e.g., Lubow, 1973). However, this "latent inhibition" effect is often reduced when preexposure occurs in a context different from that used for conditioning (see, e.g., Hall \& Channell, 1985; Swartzentruber \& Bouton, 1986). In order to minimize any differences in the levels of responding to the CS between the preexposed and nonpreexposed groups, preexposure was therefore conducted in a third context, Context $\mathrm{C}$.

\section{Method}

\section{Subjects}

The subjects were 32 male Wistar rats bred at the University of Vermont. They were 90 days old at the start of the experiment and were housed individually in standard stainless steel and wire mesh cages. The experiment was run at the same time each day during the light portion of a 15:9-h light:dark cycle. The animals were food deprived and maintained at $80 \%$ of their initial body weights throughout the experiment.

\section{Apparatus}

Three discriminably different contexts were provided by three sets of Skinner boxes housed in different rooms of the laboratory. One set consisted of four boxes measuring $26 \times 25 \times 19 \mathrm{~cm}$. The front, the back, and one sidewall were constructed of aluminum; the remaining sidewall and ceiling were made of clear acrylic plastic. The floor consisted of tubular steel bars $16 \mathrm{~mm}$ in diameter, spaced $3.2 \mathrm{~cm}$ center to center. These bars were perpendicular to the front wall, where the operant manipulanda were located. The food cup protruded from the front wall, $1 \mathrm{~cm}$ above the floor and $1 \mathrm{~cm}$ to the left of a $2.5 \times 2.5 \mathrm{~cm}$ lever. The lever's center was $5 \mathrm{~cm}$ from the right sidewall and $5 \mathrm{~cm}$ above the floor. The animals were always placed in the chamber through a door in the right sidewall. A dish containing approximately $10 \mathrm{ml}$ of Heinz white vinegar (replaced daily), positioned outside of the Skinner box immediately below the lever, provided a distinctive scent cue.

A second context, in a different room of the laboratory, was provided by four boxes measuring $24 \times 22 \times 18 \mathrm{~cm}$. The front and back walls were aluminum; the two sidewalls and ceiling were clear acrylic plastic with vertical black stripes $2 \mathrm{~cm}$ in width spaced $2.5 \mathrm{~cm}$ apart. The floor consisted of stainless steel bars $3 \mathrm{~mm}$ in diameter spaced $1.5 \mathrm{~cm}$ center to center. These bars were parallel to the front wall, where the operant manipulanda were located. A food cup was recessed in the front wall $1 \mathrm{~cm}$ above the floor and $3.5 \mathrm{~cm}$ (from its center) to the left of the sidewall. A 1-cm-wide lever protruded $4 \mathrm{~cm}$ into the box and was positioned in the center of the front wall $5 \mathrm{~cm}$ above the floor and $6 \mathrm{~cm}$ to the left of the food cup. The rats were placed in the chamber through the ceiling of the box. Approximately $1 \mathrm{~g}$ of Vicks Vaporub was smeared each day on the top of the housing surrounding the recessed food cup (outside of the Skinner box) to provide a distinctive scent cue.

In both of these two sets of four boxes, the right sidewall, composed of clear acrylic plastic, opened (via a $10 \times 12.5 \mathrm{~cm}$ opening) into a smaller side box (level with the floor) that measured $11 \times 23 \times 12.5 \mathrm{~cm}$. The top of the side box and the wall opposite the opening were constructed of clear acrylic plastic. The floor and two end walls were made of plywood painted flat black. Through a clear acrylic plastic window in the door of the sound-attenuating chamber, an experimenter could observe the rat in the side box, and through it, the Skinner box. Access to the side box was ordinarily prevented by the presence of a clear acrylic plastic door.

The third context was provided by a set of eight Skinner boxes located in two other rooms of the laboratory. Each of these boxes measured $23 \times 11 \times 13 \mathrm{~cm}$. The walls and ceiling were constructed of clear acrylic plastic. Three walls were covered on the outside with black construction paper; the front wall, which was one of the $23-\mathrm{cm}$ walls, and the ceiling were transparent. A small $(1 \times 1 \mathrm{~cm})$ food cup protruded from the back wall, $4.5 \mathrm{~cm}$ from the floor and $4.5 \mathrm{~cm}$ from the right sidewall. The animals were placed in these chambers through the ceiling of the box. The floors of four of the boxes consisted of 3-mm bars staggered in such a way that the oddnumbered bars were mounted $6 \mathrm{~mm}$ above the even-numbered bars; the distance between consecutive bars was $1.6 \mathrm{~cm}$. The bars were parallel to the narrow $(11-\mathrm{cm})$ right sidewall, on which a $1 \times 2.5 \mathrm{~cm}$ lever was centered $4.5 \mathrm{~cm}$ above the floor. A dish containing $10 \mathrm{ml}$ of a $4 \%$ coconut extract solution (replaced daily) provided a distinctive scent cue. Horizontal white stripes, $1 \mathrm{~cm}$ in width and spaced $1 \mathrm{~cm}$ apart, lined the three black walls of these boxes. In the remaining four boxes, the floors consisted of 3-mm bars, spaced $1.8 \mathrm{~cm}$ apart, that were mounted diagonally to the walls of the boxes. The lever was located near the center of the back wall, $5.5 \mathrm{~cm}$ above the floor and $8 \mathrm{~cm}$ from the food cup. In these boxes, a $2 \%$ anise solution provided the distinctive scent cue.

Forty-five-milligram Noyes food pellets (Traditional Formula) were used to reinforce the baseline in all three contexts. Illumination was provided by two $7.5-\mathrm{W}$ white incandescent bulbs, which were operated at $100 \mathrm{~V}$ ac and mounted on the ceiling of the soundattenuating chamber, 28, 27, and $25 \mathrm{~cm}$ above the floors of the different sets of boxes, respectively. The CS was a $60-\mathrm{sec}$ offset of the overhead houselights. Houselight offset was used in both of the present experiments and is hereafter referred to as the light CS. Background noise level was $65 \mathrm{~dB}$. The 0.5-sec, 0.6-mA footshock US was provided by Grason-Stadler scramblers and shock sources. Computer and electromechanical programming equipment were housed in an adjoining, centrally located room.

\section{Procedure}

Baseline training. Each daily session was $90 \mathrm{~min}$ in duration. During the first session, each rat was trained to leverpress in one of the vinegar- or Vicks-scented Skinner boxes. These two contexts, which were always completely counterbalanced, provided Contexts A and B. A food pellet was delivered for each of the first 
50 responses, after which a variable interval (VI) 90 -sec reinforcement schedule went into effect. In the next session, the procedure was repeated in a box from the other set of these contexts. In Sessions 3 and 4 , the rats were returned to the first context, where they leverpressed on the VI $90-\mathrm{sec}$ schedule of reinforcement. In Sessions 5 and 6, all animals leverpressed on the VI 90-sec schedule in one of the eight boxes from the third context, Context $\mathbf{C}$. In Sessions 7 and 8, the animals leverpressed in the remaining set of original boxes.

Preexposure. The rats were then randomly assigned to four groups $(n=8)$. For the next three sessions, all rats were placed in Context $\mathrm{C}$, in which they leverpressed on the VI 90 -sec schedule. During each of these sessions, two groups received eight nonreinforced presentations of the light CS (LI condition). The intertrial interval (ITI) was variable, with a mean of $9.5 \mathrm{~min}$. The other two groups (control condition) received equal leverpress experience in Context $\mathrm{C}$ but did not receive the light.

Conditioning. During the following four sessions, conditioning was conducted for all groups with the light CS in Context A. To equate total exposure to Contexts $A$ and $B$, these four sessions consisted of two two-session cycles. One session of each cycle was spent in Context $A$, where each of four presentations of the $60-\mathrm{sec}$ light CS terminated in the onset of the $0.5-\mathrm{sec}, 0.6-\mathrm{mA}$ footshock US. The ITI was variable, with a mean of $20 \mathrm{~min}$. During the alternate session of each cycle, the animals leverpressed on the VI schedule in Context B. Both the session of each cycle on which conditioning occurred and the actual Skinner boxes that made up these contexts were completely counterbalanced.

Testing. Extinction tests of the light were then conducted in either Context A or Context B. The light was presented four times in each of two sessions. One group from each preexposure condition, Groups LI-same and Control-same, received testing in the same context as that for conditioning, Context A. Groups LI-diff and Control-diff received testing in a different context, Context $B$. Thus, the group names designate the preexposure condition and whether extinction testing occurred in the same context as that used for conditioning or a different one.

Data analysis. Suppression to the CS was indexed in terms of suppression ratios of the form $x /(x+y)$, where $x$ represents the number of leverpresses made during the $60-\mathrm{sec} \mathrm{CS}$ and $y$ the number of leverpresses made during the $60-\mathrm{sec}$ immediately preceding the CS (the pre-CS period). A suppression ratio of 0 indicates complete response suppression during the $\mathrm{CS}$, whereas a ratio of 0.5 indicates no suppression of responding during the CS. The suppression ratios were analyzed with analyses of variance (ANOVAs); simple effects were evaluated with the standard methods described by Howell (1987, pp. $431 \mathrm{ff}$ ). Leverpress rates during the pre-CS periods were also routinely analyzed with ANOVAs identical to those used to analyze the suppression ratios. All statistical tests had a rejection criterion of $p<.05$.

\section{Results and Discussion}

The left side of Figure 1 presents two-trial blocks of suppression to the light for the four groups during the conditioning phase. Latent inhibition, resulting from the 24 trials of preexposure to the light in Groups LI-same and LI-diff, was demonstrated by weaker responding in the LI groups, compared to Groups Control-same and Control-diff. This impression was confirmed by a three-way ANOVA examining preexposure condition, test context, and trial blocks. The ANOVA revealed significant main effects of preexposure condition $[F(1,28)=8.89]$ and trial blocks $[F(3,84)=34.92]$. No other main effects or interactions approached significance $(F \mathrm{~s}<1)$. This experiment did not include a group that received preexposure in the same con-

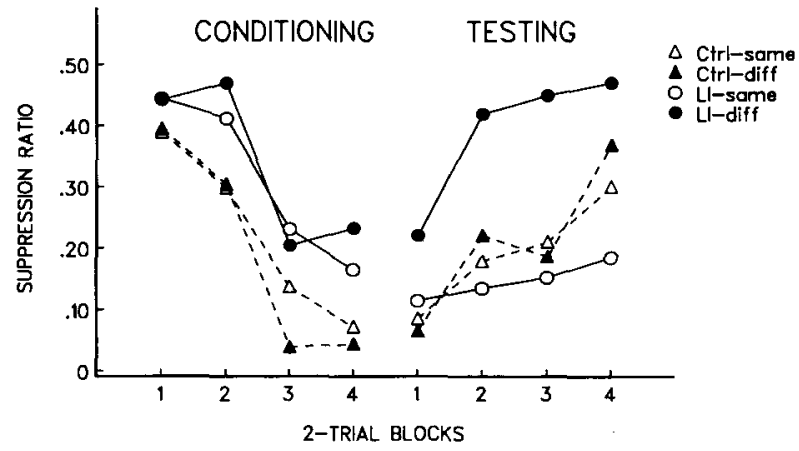

Figure 1. Two-trial blocks of suppression to the light during conditioning and extinction testing in Experiment 1. Same and diff groups received testing in either the same context as that used for conditioning or a different context, respectively. The LI groups received nonreinforced exposure to the light prior to conditioning.

text as that used for conditioning; however, prior research has demonstrated that a context switch consistently reduces, but does not always eliminate, the latent inhibition effect (e.g., Lovibond et al., 1984, Experiment 2).

Pre-CS leverpress rates for Groups LI-same, LI-diff, Control-same, and Control-diff were 18.2, 17.4, 20.1, and 15.0 , respectively. There were no group differences or interactions $(F \mathrm{~s}<1.21)$.

The results of most interest, those from the extinction test trials in either the same or the different context from conditioning, are presented on the right side of Figure 1. In agreement with previous research (e.g., Bouton \& King, 1983; Lovibond et al., 1984), Groups Control-same and Control-diff showed equal levels of responding throughout the test, regardless of the context in which testing occurred. On the other hand, Group LI-diff showed weaker responding than did Group LI-same, which remained relatively suppressed throughout the eight trials of extinction.

These observations were confirmed in an ANOVA that revealed significant main effects of test context $[F(1,28)$ $=4.75]$ and trial blocks $[F(3,84)=22.45]$, and marginally significant interactions of preexposure condition $x$ test context $[F(1,28)=3.61, p=0.68]$ and trial blocks $\times$ test context $[F(3,84)=2.70, p=.051]$. A priori simple effects tests were used to examine the influence of the test-context identity on responding in the $\mathrm{LI}$ and control groups collapsed across trial blocks. These analyses revealed a significant effect of test context only in the LI condition; Group LI-diff showed significantly less suppression than did Group LI-same $[F(1,28)=8.32]$, whereas Groups Control-diff and Control-same did not differ $[F(1,28)<1]$. Furthermore, although differences between the LI and control groups during acquisition complicate individual comparisons between these groups during the test, simple effects tests revealed a reliable effect of preexposure only when testing occurred in a different context; Group LI-diff showed reliably less suppression than did Group Control-diff $[F(1,28)=4.55]$, whereas Groups LI-same and Control-same did not differ $[F(1,28)<1]$. 
The mean pre-CS leverpress rates were 21.6, 27.2, 25.8, and 19.6, for Groups LI-same, LI-diff, Controlsame, and Control-diff, respectively. An ANOVA identical to that conducted for suppression revealed no main effects or interactions involving the group factors $(F \mathrm{~s}<2.1)$.

The finding that Groups Control-same and Control-diff responded at the same level throughout extinction testing is consistent with other research in which conditioned suppression is seldom affected by a context change following simple conditioning. However, the difference between Groups LI-same and LI-diff suggests that conditioned responding is influenced by the test context when conditioning is preceded by nonreinforced CS exposure in a different context.

The apparent weaker responding in Group LI-diff than in Group Control-diff during testing may contrast with data reported by Lovibond et al. (1984, Experiment 2), in which conditioning generalized across contexts even after latent inhibition training. In their experiment, two groups were preexposed to two CSs, each in a separate context. Following conditioning of both CSs in contexts that were either the same as or different from the contexts of preexposure, both groups were tested in the nonconditioning context. Thus, like the present Group LI-diff, these latent inhibition groups received a context switch following conditioning. However, unlike the responding of the present group, their responding during extinction was no different from that in a group that had not received CS preexposure. Thus, conditioning appeared to transfer across contexts. There are several procedural differences between that experiment and the present one. We would note that, because the experiments investigated different issues, the comparisons we emphasized here (between the LI groups and between the control groups tested in conditioning or alternate contexts), are not available in the Lovibond et al. design. In addition, procedures in which two CSs are conditioned in separate contexts prior to a context switch have sometimes produced different outcomes. For example, using such a procedure, Kaye and Mackintosh (1990) found enhanced suppression to a simple excitor following a context switch, rather than merely no change in performance. No such effect was found in the present control groups. With the present methods, CS preexposure appears to increase context sensitivity of conditioned responding.

\section{EXPERIMENT 2}

The results of Experiment 1 are consistent with the possibility that sensitivity to contextual cues following conditioning may depend on the nature of the animal's prior experience with the CS. However, because preexposure was conducted in a different context from that used for conditioning, it is possible that the increase in context sensitivity depended on the animal's having received the different CS treatments in different contexts. Experiment 2 was designed to examine the importance of the preexposure context in the context sensitivity of condi- tioned responding. Prior to conditioning in Context A, two pairs of groups received CS exposure in either Context A or Context C. Within each pair of groups, testing consisted of extinction trials in a context that was either the same as (Context A) or different from (Context B) that used for conditioning. If the effect of CS preexposure on context sensitivity is independent of the preexposure context, responding should be sensitive to the context for both pairs of groups.

The design also provided the opportunity to examine an aspect of latent inhibition that has not received much attention. Many associative learning models imply that the weaker excitatory conditioning that results from CS preexposure might produce stronger context-US associations during conditioning (e.g., Mackintosh, 1975; Pearce \& Hall, 1980). Manipulations that reduce acquisition to a CS might facilitate the conditioning of cues present in compound with it. Because latent inhibition is often reduced when the context is changed between preexposure and conditioning (see, e.g., Hall \& Channell, 1985; Swartzentruber \& Bouton, 1986), we tested this possibility by comparing the conditioning of context excitation in groups that received preexposure in either the same context as that used for conditioning or a different one. Importantly, in the present design, exposure to both the CS and contexts was equated across groups. The groups differed only with respect to whether or not CS preexposure had occurred in the conditioning context.

The experiment was conducted in two replications that differed in several minor respects. In the first replication, there were 24 preexposure trials; we monitored both the acquisition of context excitation and excitation to the CS during the conditioning phase. However, the results revealed only small differences in the conditioning rate between the groups that received preexposure in the same context as that used for conditioning and those that received preexposure in a different context. Because a context-preference procedure was introduced and used to measure context excitation at the beginning of each conditioning session (Bouton \& King, 1983), it was possible that all groups perceived the conditioning context as different from the preexposure context (which did not involve context-preference tests). In an effort to increase latent inhibition and the effect of the context switch following preexposure, the second replication involved 36 preexposure trials and no context-preference testing.

\section{Method}

\section{Subjects and Apparatus}

The subjects were 64 ( 32 in each replication) male Wistar rats from the same stock as before, between 90 and 130 days old at the start of the experiments. The apparatus, housing, and maintenance conditions were the same as in Experiment 1.

\section{Procedure}

Baseline training. Baseline training followed the procedure used in Experiment 1, except as noted. In the first replication, the doors to the side boxes were left open on the final session of baseline training in Contexts A and B to allow exploration of the side boxes. 
The second replication included a third session of shaping in Context $\mathrm{C}$, to provide equal exposure to the three contexts. In both replications, the sets of boxes that provided Contexts $A$ and $B$ (but not Context C) were counterbalanced.

Preexposure. Following the baseline training phase of each replication, the rats were randomly assigned to four groups $(n=8)$. The preexposure phase consisted of three three-session cycles similar to the conditioning cycles of Experiment 1. The first two sessions of each cycle were spent leverpressing in Contexts $A$ and $C$ (order counterbalanced within groups). Eight nonreinforced presentations of the light occurred during one of these two sessions. For two groups, the light was presented in Context C; for the other two groups, the light was presented in Context A. In the second replication, the number of light presentations per cycle was increased to 12 , with an average ITI of $6 \mathrm{~min}$. For all groups, the third session of each cycle was spent leverpressing in Context B.

Conditioning. During the next four sessions, the light was conditioned in Context $\mathbf{A}$. To equate total exposure to Contexts $\mathbf{A}$ and $B$, these four sessions consisted of two two-session cycles. One session of each cycle (counterbalanced) was spent in Context $A$, in which the light was paired four times with the US with a mean ITI of 20 min. The alternate session of each cycle was spent leverpressing in Context B.

Testing. Responding to the light during extinction was then assessed in either Context A or Context B. The light was presented alone four times in each of two sessions. One group from each preexposure condition, Groups A-same and $\mathrm{C}$-same, received extinction in the same context as that used for conditioning, Context $\mathrm{A}$. Groups A-diff and C-diff received extinction in a different context, Context B. The group names thus designate both the context of preexposure and whether extinction occurred in either the same context as that used for conditioning or a different context.

Behavioral observation. In the first replication, each session of conditioning included context-preference tests in order to assess possible group differences in context conditioning. For the first 6 min of each session, the rats were allowed to choose between the interior of the Skinner box, where they could earn food pellets on the VI schedule, and the adjacent side box. With a time-sampling technique, each rat's location was recorded every $12 \mathrm{sec}$ for a total of 30 samples. At the conclusion of the test, the door between the Skinner box and the side box was replaced for the remainder of the session. Each rat's preference was expressed as a contextpreference score, which consisted of the number of samples recorded as "in" the Skinner box (out of a possible 30). Low scores indicate contextual fear.

\section{Results and Discussion}

There were no significant effects involving replication in any phase; the reported ANOVAs therefore collapsed across this factor. The left side of Figure 2 presents twotrial blocks of suppression to the light during conditioning. Suppression in Groups A-same and A-diff was weaker than in Groups $\mathrm{C}$-same and $\mathrm{C}$-diff, which suggests that a change of context between preexposure and conditioning reduced the interfering effects of CS preexposure. A three-way ANOVA examining the contexts of preexposure and testing across trial blocks revealed significant effects of the preexposure context $[F(1,60)=10.88]$ and trial blocks $[F(3,180)=52.99]$. No other main effects or interactions were significant $(F \mathrm{~S}<1.24)$. Mean pre-CS leverpressing rates for Groups A-same, A-diff, C-same, and C-diff were 13.6, 16.4, 17.1, and 16.7, respectively. There were no significant main effects or interactions involving the group factors $(F \mathrm{~s}<1.84)$.

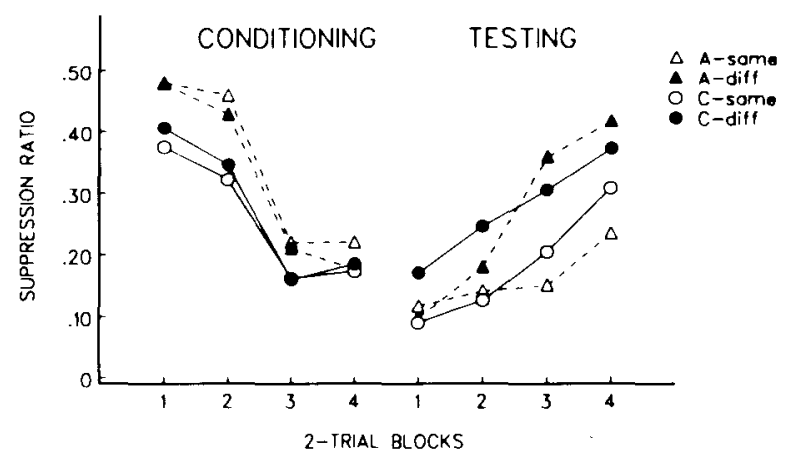

Figure 2. Two-trial blocks of suppression to the light during conditioning in Context $A$ and extinction testing in Experiment 2. Same and diff groups received testing in either the same context (A) as that used for conditiong or a different context (B), respectively. A and $\mathbf{C}$ refer to the context of preexposure.

The data of most interest, those from the test sessions conducted in either the same context as that used for conditioning or a different context are presented on the right side of Figure 2. In general, under both preexposure context conditions, the groups that were tested in a different context showed less suppression than did the groups tested in the conditioning context. Thus, context sensitivity was revealed, regardless of the relationship between the preexposure and conditioning contexts. This impression was confirmed by an ANOVA that revealed a significant main effect of the test context $[F(1,60)=6.40]$. The analysis also revealed a main effect of trial blocks $[F(3,180)=$ 40.15], and significant interactions of test context $\times$ trial blocks $[F(3,180)=3.28]$ and preexposure context $\times$ test context $\times$ trial blocks $[F(3,180)=3.96]$. No other main effects or interactions approached significance $(F \mathbf{S}<1)$. A test of the simple interaction effects examining the threeway interaction revealed a change in suppression over trial blocks in Group A-diff as opposed to A-same $[F(3,180)$ $=6.92]$. The difference between Groups C-same and C-diff did not change over trials $[F(3,180)<1]$. This difference in the pattern of responding suggests that the identity of the preexposure context might affect the manner in which context sensitivity is exhibited.

Mean pre-CS rates were $16.8,19.2,20.8$, and 18.3, for Groups A-same, A-diff, C-same, and C-diff, respectively. There were no significant main effects or interactions involving the group factors $(F \mathrm{~s}<2.0)$.

Context-preference scores measuring excitation in Context A during the first replication were subjected to an ANOVA in which context preference was examined as a function of the preexposure and test contexts across the two conditioning sessions. It was conceivable that slower conditioning for the groups that received preexposure in the conditioning context might have been accompanied by more excitation conditioned to the context. The contextpreference data, however, revealed no evidence of context excitation or of any group differences in context preference as a result of conditioning. In the first session 
of conditioning, prior to the delivery of any USs, the mean context-preference scores for Groups A-same, A-diff, C-same, and C-diff were 15.0, 16.6, 16.7, and 17.0, respectively. On the second session, the scores were 14.4 , $15.2,15.4$, and 18.6. There were no significant main effects or interactions $(F \mathrm{~s}<1)$.

These results again demonstrate that conditioned responding to a preexposed CS is sensitive to the identity of the test context. Ordinarily, with multiple-trial conditioning procedures, conditioned suppression is not attenuated when the CS is tested in an alternate context following conditioning (e.g., Experiment 1; Bouton \& King, 1983; Grahame et al., 1990; Hall \& Honey, 1989; Kaye \& Mackintosh, 1990; Kaye et al., 1987; Lovibond et al., 1984; Swartzentruber \& Bouton, 1986). However, as in Experiment 1, sensitivity to the context was apparent here in animals that received CS exposure prior to conditioning. In addition, the present results suggest that the enhancement of context sensitivity does not depend on preexposure and conditioning occurring in different contexts.

\section{GENERAL DISCUSSION}

These experiments examined the effects of CS preexposure on the context sensitivity of conditioned responding. In Experiment 1, when the CS was novel at the outset of conditioning, conditioned responding was unaffected by the context in which extinction occurred. This result replicates the results of many previous experiments in which conditioning generalized strongly across contexts (e.g., Bouton \& King, 1983; Grahame et al., 1990; Hall \& Honey, 1989; Kaye \& Mackintosh, 1990; Kaye et al., 1987; Lovibond et al., 1984; Swartzentruber \& Bouton, 1986), and it suggests that simple excitatory conditioning can be relatively insensitive to contextual stimuli. However, for groups that had received prior nonreinforced CS exposure, conditioned responding was sensitive to the identity of the extinction context. For these groups, responding was weaker when extinction was conducted outside of the conditioning context. Experiment 2 further demonstrated that conditioning is sensitive to the context when preexposure trials occur in the same context as that used for conditioning.

Several views might account for the present pattern of results. One explanation might emphasize possible differences in the levels of context excitation that resulted from conditioning. If preexposure decreases the associability of the CS (e.g., Mackintosh, 1975; Pearce \& Hall, 1980; Wagner, 1981), the ability of the CS to compete with the context for associative strength might correspondingly be weakened. In groups with CS preexposure, then, the conditioning context could have become especially excitatory. If context excitation enhances responding to the CS, the preexposed groups might show a greater response decrement when testing occurs in a less excitatory context. However, neither baseline (pre-CS) response rates nor the context-preference tests (Experiment 2) confirmed the kinds of differences in context excitation that are suggested by this analysis. Furthermore, there is surprisingly little evidence in the literature to support the view that context excitation actually enhances responding to the $C S$ in the conditioned suppression preparation. For example, when CSs are tested in contexts made demonstrably excitatory by separate exposure to the US, there is typically no enhancement of suppression to a simple CS (see, e.g., Bouton, 1984; Bouton \& King, 1986; Randich \& Ross, 1984). It thus seems unlikely that differential context excitation could have contributed in a major way to the present results.

A second possibility is that weaker CS-US representations might be easier to disrupt with a context switch. Preexposure might reduce the associative strength acquired during conditioning because it reduces attention or CS processing (see, e.g., Mackintosh, 1975; Pearce \& Hall 1980; Wagner, 1981); alternatively, the memory of preexposure might interfere with the retrieval of the CS-US association (see, e.g., Bouton, 1991; Spear, 1981). Either mechanism might result in a weaker, more disruptable CS-US representation. In agreement with this possibility, Hall and Honey (1990, Experiment 3) found that a context change attenuated conditioned suppression if rats were given only a single conditioning trial; conditioning with multiple trials generalized across contexts. Although in their experiment suppression was strongest in the one-trial groups because of the use of a stronger US in these groups, it is nevertheless conceivable that the CS-US association is less memorable after a single conditioning trial than after multiple trials. Weak memories might be more susceptible to disruption by a change of context.

Finally, Bouton and Bolles (1985) and Bouton and King (1986) suggested that the meaning of the CS might be "ambiguous" when it has been associated with different outcomes such as reinforcement and nonreinforcement. According to this view, the preexposed groups could have stored representations corresponding to reinforcement and nonreinforcement of the CS (see also Spear, 1981). The context would then be important in resolving the resulting ambiguity by retrieving the corresponding representation. Although this perspective suggests that the context may be important when a CS is associated with mixed outcomes, it does not provide a mechanism that would explain the present data directly. One possibility would be to follow a lead from the memory retrieval literature. Baddeley (1982) suggested that contextual stimuli become important retrieval cues only when the learning task encourages the subject to "integrate" contextual information with a target memory (see also Eich, 1985). When the CS changes "meaning" in the conditioning phase of a latent inhibition experiment, the context may no longer be an incidental stimulus, and the animal may be more likely to encode contextual information along with the CS-US association. This approach is speculative; however, the present data suggest that responding to a CS may be more sensitive to contextual features when the CS is associated with different outcomes in different phases of the experiment. 
In any case, the present experiments have uncovered a variable that appears to influence the extent to which conditioned responding is sensitive to contextual stimuli. The results suggest that the role of the context depends at least partly on the nature of the prior conditioning history of the CS. Although there is little empirical evidence that might distinguish an explanation based on the strength of conditioning from one emphasizing a memory retrieval process, the present results help elucidate the conditions under which contexts might acquire control of responding to simple excitatory CSs.

\section{REFERENCES}

Baddeley, A. D. (1982). Domains of recollection. Psychological Review, 89, 708-729.

Balsam, P. D., \& Tomie, A. (1985). Context and learning. Hillsdale, NJ: Erlbaum.

Bouton, M. E. (1984). Differential control by context in the inflation and reinstatement paradigms. Jourmal of Experimental Psychology: Animal Behavior Processes, 10, 56-74.

Bouton, M. E. (1991). Context and retrieval in extinction and in other examples of interference in simple associative learning. In $\mathrm{L}$. W. Dachowski \& C. F. Flaherty (Eds.), Current topics in animal leaming: Brain, emotion, and cognition (pp. 25-53). Hillsdale, NJ: Erlbaum.

Bouton, M. E., Bolles, R. C. (1979). Contextual control of the extinction of conditioned fear. Learning \& Motivation, 10, 445-466.

Bouton, M. E., Bolles, R. C. (1985). Contexts, event-memories, and extinction. In P. D. Balsam \& A. Tomie (Eds.), Context and learing (pp. 133-166). Hillsdale, NJ: Erlbaum.

Bouton, M. E., KING, D. A. (1983). Contextual control of the extinction of conditioned fear: Tests for the associative value of the context. Joumal of Experimental Psychology: Animal Behavior Processes, 9, 248-265.

Bouton, M. E., King, D. A. (1986). Effect of context on performance to conditioned stimuli with mixed histories of reinforcement and nonreinforcement. Joumal of Experimental Psychology: Animal Behavior Processes, 12, 4-15.

Bouton, M. E., a Peck, C. A. (1989). Context effects on conditioning, extinction, and reinstatement in an appetitive conditioning preparation. Animal Learning \& Behavior, 17, 188-198.

Bouton, M. E., \&WARTzentruber, D. (1986). Analysis of the associative and occasion-setting properties of contexts participating in a Pavlovian discrimination. Joumal of Experimental Psychology: Animal Behavior Processes, 12, 333-350.

EıCH, E. (1985). Context, memory, and integrated item/context imagery. Journal of Experimental Psychology: Leaming, Memory, \& Cognition, 11, 764-770.
Grahame, N. J., Hallam, S. C., Geier, L., Miller, R. R. (1990). Context as an occasion setter following either CS acquisition and extinction or CS acquisition alone. Leaming \& Motivation, 21, 237-265.

Hall, G. . Channell, S. (1985). Differential effects of contextual change on latent inhibition and on the habituation of an orienting response. Journal of Experimental Psychology: Animal Behavior Processes, 11, 470-481.

HALL, G., Honey, R. C. (1989). Contextual effects in conditioning, latent inhibition, and habituation: Associative and retrieval functions of contextual cues. Journal of Experimental Psychology: Animal Behavior Processes, 15, 232-241.

HaLL, G., Honey, R. C. (1990). Context-specific conditioning in the conditioned emotional response procecture. Jowmal of Experimental Psychology: Animal Behavior Processes, 16, 271-278.

HowELL, D. C. (1987). Statistical methods for psychology. Boston: Duxbury.

KAYE, H., MACKIntosh, N. J. (1990). A change of context can enhance performance of an aversive but not of an appetitive conditioned response. Quarterly Joumal of Experimental Psychology, 42B, 113-134.

Kaye, H., Preston, G. C., Szabo, L., Druiff, H., Mackintosh, N. J. (1987). Context specificity of conditioning and latent inhibition: Evidence for a dissociation of latent inhibition and associative interference. Quanerly Journal of Experimental Psychology, 39B, 127-145.

Lovibond, P. F., Preston, G. C., « Mackintosh, N. J. (1984). Context specificity of conditioning, extinction, and latent inhibition. Jowmal of Experimental Psychology: Animal Behavior Processes, 10, 360-375.

Lubow, R. E. (1973). Latent inhibition. Psychological Bulletin, 79, 398-407.

MACKINTOSH, N. J. (1975). A theory of attention: Variations in the associability of stimuli with reinforcement. Psychological Review, 82, 276-298.

Pearce, J. M., Hall, G. (1980). A model for Pavlovian learning: Variations in the effectiveness of conditioned but not of unconditioned stimuli. Psychological Review, 87, 532-555.

RANDICH, A., Ross, R. T. (1984). Mechanisms of blocking by contextual stimuli. Leaming \& Motivation, 15, 106-117.

SPEAR, N. E. (1981). Extending the domain of memory retrieval. In N. E. Spear \& R. R. Miller (Eds.), Information processing in animals: Memory mechanisms (pp. 341-378). Hillsdale, NJ: Eribaum.

Swartzentruber, D., Bouton, M. E. (1986). Contextual control of negative transfer produced by prior CS-US pairings. Leaming \& Motivation, 17, 366-385.

W AGNER, A. R. (1981). SOP: A model of automatic memory processing in animal behavior. In N. E. Spear \& R. R. Miller (Eds.), Information processing in animals: Memory mechanisms (pp. 5-47). Hillsdale, NJ: Erlbaum.

(Manuscript received June 26, 1991; revision accepted for publication November 19, 1991.) 